\title{
Anticoncepción hormonal en mujeres jóvenes con enfermedades reumatológicas
}

\author{
Danisa Ivanovic-Zuvic S. ${ }^{a}$, Carolina Pastene $S^{1}{ }^{1}$, Marcela Menéndez A. ${ }^{1}$, Claudia Zajer A. ${ }^{1}$ \\ ${ }^{1}$ Unidad de Ginecología Infanto Juvenil, División de Obstetricia y Ginecología, Facultad de Medicina Pontificia Universi- \\ dad Católica de Chile.
}

a Alumna, Escuela de Medicina, Pontificia Universidad Católica de Chile.

\section{RESUMEN}

Antecedentes: Las enfermedades reumatológicas (ER) se presentan con mayor frecuencia en mujeres en edad fértil. Es durante este período que muchas mujeres consultan por trastornos de los flujos rojos, dismenorrea y prevención del embarazo. Objetivo: Realizar una puesta al día del uso de anticoncepción hormonal (AH) en mujeres jóvenes con ER, especialmente en las portadoras de lupus eritematoso sistémico (LES) y artritis reumatoide (AR). Método: Se revisó la literatura en Pubmed para los términos AH en ER, con especial énfasis en guías clínicas y ensayos clínicos aleatorizados. Resultados: El uso de AH en pacientes con LES inactivo o con actividad leve no ha mostrado un empeoramiento de la enfermedad. Tampoco existe evidencia categórica que demuestre un mayor riesgo trombótico en aquellas con anticuerpos antifosfolípidos negativos, para las cuales se recomienda el uso de cualquier tipo de $\mathrm{AH}$. En pacientes con AR no se ha demostrado asociación del uso de AH y aumento de la actividad de la enfermedad. Además la interacción farmacológica entre $\mathrm{AH}$ y medicamentos reumatológicos es mínima. Existe escasa evidencia del uso de AH en mujeres menores de 18 años con ER. Conclusión: Basado en la evidencia disponible las pacientes con ER con anticuerpos antifosfolípidos negativos podrán utilizar AH según los criterios de la OMS, lo que abre las puertas a mayores alternativas terapéuticas y prevención de embarazo para estas adolescentes y mujeres jóvenes con ER.

\section{PALABRAS CLAVE: Anticoncepción, lupus eritematoso sistémico, artritis reumatoide, enfermedades reumatológicas, trombosis}

\section{SUMMARY}

Background: Rheumatologic diseases (RD) are most common in women of childbearing age. It is during this period that many women consult for menstrual disorders, dysmenorrhea and pregnancy prevention. Objective: To update the use of hormonal contraception $(\mathrm{HC})$ in young women with RD. Method: Literature was reviewed in PubMed for $\mathrm{HC}$ and $\mathrm{RD}$ terms, with special emphasis on clinical guidelines and randomized clinical trials. Results: The use of $\mathrm{HC}$ in patients with inactive or mild activity systemic lupus erythematosus has not shown a worsening of the disease. Also, there is no evidence demonstrating greater thrombotic risk in those with negative antiphospholipid antibodies, for which the use of any type of $\mathrm{HC}$ is recommended. In patients with rheumatoid arthritis an association between use of $\mathrm{HC}$ and increases disease activity has not been demonstrated. In addition, there is minimal drug interaction between $\mathrm{HC}$ and rheumatologic drugs. There is little evidence of the use of $\mathrm{HC}$ in women less than 18 years with RD. Conclusion: Based on the available evidence the patients with RD and negative antiphospholipid antibodies could use $\mathrm{HC}$ according 
to WHO criteria, which leads to greater therapeutic alternatives and pregnancy prevention for these adolescents and young women with RD.

\section{KEY WORDS: Contraception, systemic lupus erythematosus, rheumatoid arthritis, rheumatologic diseases, thrombosis}

\section{INTRODUCCIÓN}

Las enfermedades autoinmunes que comprometen las articulaciones y el tejido músculo-esquelético, tales como el lupus eritematoso sistémico (LES), la artritis reumatoide (AR), el síndrome de Sjöegren, la esclerosis múltiple, la miositis y la dermatomiositis, afectan principalmente a la mujer en edad fértil. Debido a este patrón epidemiológico, se ha estudiado una posible asociación entre las enfermedades reumatológicas (ER) y las hormonas sexuales femeninas. En este contexto, se ha demostrado que los estrógenos pueden promover la maduración de los linfocitos $\mathrm{T}$ helper, potenciar la actividad fagocítica y de presentación de antígeno de los macrófagos, y activar a los linfocitos B (1), lo que favorecería un flare up de las enfermedades dependientes de la inmunidad celular. Por otro lado, los andrógenos han demostrado un rol protector $(2,3,4)$.

Esta asociación entre las hormonas sexuales femeninas y las ER ha sustentado la precaución constante del uso de la anticoncepción hormonal $(\mathrm{AH})$ en pacientes con $\mathrm{ER}$, por el riesgo de activar o empeorar el curso de estas enfermedades. Además, en múltiples oportunidades se ha desincentivado el uso de $\mathrm{AH}$ por las duda en torno a la seguridad de estos métodos, especialmente por los riesgos trombóticos reportados.

Esto ha hecho que mujeres con ER no utilicen métodos anticonceptivos, o solo utilicen métodos de barrera para la prevención del embarazo, lo que las expone a gestaciones no planificadas que pueden coincidir con el uso de medicamentos con efectos teratogénicos, tales como el metotrexate, ciclofosfamida o micofenolato, en cuyo caso la prevención del embarazo se hace imprescindible. A su vez, el uso de $\mathrm{AH}$ en adolescentes y jóvenes sin actividad sexual se hace necesario como herramienta terapéutica en el manejo de la metrorragia y la dismenorrea.

Es por estos motivos que el ginecólogo deberá estar familiarizado con la mejor alternativa de $\mathrm{AH}$ guiado por la mejor evidencia disponible, para cada caso, brindando la mayor efectividad con un mínimo de efectos adversos. Estos deben ser indicados a la usuaria y a su pareja/familia, a través de una consejería en salud sexual y reproductiva, que nos permitirá dar mejores soluciones a la calidad de vida de nuestras pacientes.
El objetivo de este artículo es revisar la evidencia existente en torno a la seguridad del uso de la $\mathrm{AH}$ en pacientes jóvenes con ER, con especial énfasis en las portadoras de LES y AR, para facilitar la toma de decisiones en torno a la salud sexual y reproductiva de éstas.

\section{LUPUS ERITEMATOSO SISTÉMICO Y ANTICON- CEPCIÓN}

EI LES es una enfermedad autoinmune de causa desconocida que afecta a múltiples órganos y sistemas. La incidencia del LES es de un 5,7-19,3 por 100.000 mujeres (5), y es 10 veces más frecuente que en el hombre (6). Generalmente se presenta después de la menarquía y antes de la menopausia. Varios estudios han mostrado un riesgo de 2 a 3 veces mayor de activación de la enfermedad durante el embarazo $(7,8)$. EI LES es una condición mediada principalmente por citoquinas tipo Th2. En el embarazo, los estrógenos cambiarían el balance de la producción de citoquinas Th1/Th2, a favor de las citoquinas Th2. Argumento central que propone a los estrógenos como agravantes de la enfermedad durante el embarazo (1). Además, las pacientes con LES moderado o severo presentan mayores complicaciones obstétricas, tales como aborto, preeclampsia, parto prematuro, muerte materna y fetal $(9,10)$. Es por esto que las pacientes con LES requieren un control ginecológico establecido, consejería en salud sexual y reproductiva, programación de los embarazos en el período de quiescencia de actividad de la enfermedad y un control multidisciplinario estricto durante la gestación.

Actividad del LES y uso de anticoncepción. La actividad del LES se evalúa con diferentes métodos. Una de las más utilizadas es la escala SLEDAI (Systemic Lupus Erythematosus Disease Activity), la cual comprende 24 parámetros clínicos y de laboratorio (11). Se otorga mayor puntaje a mayor actividad. En general no existe un consenso sobre el valor de corte que defina a la enfermedad leve, moderada o severa, pero en la mayoría de los artículos se considera un brote cuando hay un aumento de 3 a 11 puntos desde el último control, y un brote severo si hay un aumento de 12 o más puntos desde el último control. A su vez, se han descrito tres patrones de la actividad de la enfermedad en el tiempo (12): a. Crónico activo (actividad persistente 
en un año), b. Remisión (sin actividad clínica en un año) y c. Remisión-exacerbación o brotes.

Durante muchos años se temió el uso de anticoncepción con estrógenos, basado en dos estudios observacionales de los años 1982 y 1991, los cuales concluyeron una asociación entre el uso de $\mathrm{AH}$ combinada y el deterioro de la enfermedad, por lo que se desincentivó el uso de estrógenos en mujeres con LES (13 -14). El año 2005 este concepto cambió con la aparición de dos estudios clínicos randomizados (ECR).

En el ECR de Petri y cols (15) se evaluó el uso de anticoncepción oral combinado (ACO) en esquema trifásico, con 35 ug etinilestradiol y noretisterona vs placebo. Se incluyeron 183 pacientes entre 18 a 39 años, con LES inactivo o activo estable (score SELENA-SLEDAI con rango entre 0 a 12, promedio 3,01 para ACO y 3,09 para placebo), se excluyeron pacientes que tenían niveles de anticuerpos anticardiolipinas o anticoagulante lúpico moderado a severo. Durante el seguimiento a 12 meses se observó que ambos grupos no presentaron diferencias significativas en cuanto a brotes severos de lupus $(7,7 \%$ ACO vs $7,6 \%$ placebo), tasa de brotes severos a los 12 meses y de brotes leves o moderados (69\% ACO vs $60 \%$ placebo).

El segundo estudio de Sanchez-Guerrero y cols (16) evaluó el uso de ACO con 30 ug de etinilestradiol y 150 mcg levonogestrel vs progestágeno oral (POP) de $30 \mathrm{mcg}$ levonogestrel vs dispositivo intrauterino (DIU) de cobre. Se randomizaron 162 mujeres con LES, entre 21 a 33 años, independiente de sus niveles de anticuerpos anticardiolipinas. Se excluyeron mujeres con SLEDAI >30 (con un rango de score de SLEDAI de 0 a 26 y promedio de score de 6,1 para el grupo de ACO, 6,4 para POP, y 5,0 para DIU). Durante el seguimiento a 12 meses no se observaron diferencias significativas entre los tres grupos en cuanto a la actividad global de la enfermedad (SLEDAI-AUC), máximo score de SLEDAI, cambio en score de SLEDAI, incidencia de brotes, tiempo transcurrido hasta el primer brote, ni en el empleo de medicamentos.

Una revisión sistemática (RS) del año 2009 evaluó el uso de $\mathrm{AH}$ en pacientes con LES inactivo o activo estable (17). Incluyó 13 estudios; 2 de los cuales eran los ECR ya descritos. Esta RS indicó que el uso de $\mathrm{AH}$ oral -tanto con estrógeno mas progesterona como progesterona sola- no aumentaría la activación, ni agravaría la condición de mujeres con LES inactivo o activo estable.

Riesgo trombótico en LES y anticoncepción. El estrógeno promueve la síntesis de varios factores de coagulación trombogénicos y disminuye el efecto de algunos factores anticoagulantes. El uso de AH combinada está asociado al desarrollo de tromboembolismos en población general (18). Se describen incidencias de 1:10.000 a 1:100.000 dependiendo de la edad, en comparación al riesgo de eventos trombóticos en población general sin uso de $\mathrm{AH}$ de <1:100.000 (19). Los anticonceptivos de progestinas son menos trombogénicos, pero se ha observado que el uso de ACO con desogestrel tiene mayor riesgo de trombosis venosa que con el uso de norgestrel o levonogestrel $(20,21)$. Las mujeres con LES tienen un riesgo aumentado de trombosis en comparación a la población general, con reportes de 1:10 a 1:100 (22). Su origen es multifactorial; se han identificado como factores de riesgo: estrógenos, tabaquismo, edad, actividad de la enfermedad, dosis de glucocorticoides, duración de la enfermedad, etnia, dislipidemia, obesidad, insuficiencia venosa, vasculitis, síndrome nefrótico y significativamente los anticuerpos antifosfolípicos positivos (23). La presencia de anticuerpos antifosfolípidos (anticoagulante lúpico, anticuerpo anticardiolipina y anticuerpo anti- $\beta 2$-glicoproteina) aumenta el riesgo de trombosis tanto arterial como venosa $(16,24,25)$, y las pacientes con LES tienen mayor riesgo de trombosis con anticuerpos positivos en comparación a las que no los tienen (trombosis venosa: OR=5,6; IC95\% 3,8-8,3; trombosis arterial: $\mathrm{OR}=2,2 ; \mathrm{IC} 95 \%$ 1,5-3,1) (26).

Se ha observado una asociación positiva de pacientes con anticuerpos antifosfolípidos y el uso de $\mathrm{AH}$ en eventos trombóticos. En el estudio de casos y controles de Chopra y cols (27) se evaluaron 157 pacientes (131 mujeres y 26 hombres) con anticuerpos antifosfolípidos positivos. De ellos, 69 presentaron historia de trombosis y 88 no. Se observó que el grupo con historia de trombosis reportaba el uso más frecuente de $\mathrm{AH}$ (se desconoce tipo y dosis), pero sin significancia estadística.

Otro estudio de casos y controles de Choojitarom y cols (28) evaluó a 65 pacientes con LES y anticuerpos antifosfolípidos positivos. Se observó que de las 3 pacientes que usaron ACO todas tuvieron eventos trombóticos, a diferencia de las 23 de 62 que no usaron ACO. Se desconoce el rol específico que podría tener la $\mathrm{AH}$ en el aumento del riesgo trombótico en pacientes con anticuerpos antifosfolípidos positivos. Pero en base a esto, el uso de $\mathrm{AH}$ con estrógenos ha sido contraindicado dado el alto riesgo trombótico que conlleva asociado a esta condición (29).

Para pacientes con LES y anticuerpos antifosfolípidos negativos, la evidencia es aún más escasa. Hasta el día de hoy no se cuenta con estimaciones adecuadas de la magnitud de riesgo de trombosis asociado a la $\mathrm{AH}$ en pacientes con LES.

Otras complicaciones. Recientemente Cravioto y cols evaluaron en un ECR los efectos adversos asociados al uso de distintos métodos anticonceptivos en LES (30). Durante un año siguió a 162 mujeres menores de 40 años que fueron asignadas al uso de ACO (30 ug etinilestradiol y 150 mcg levonogestrel), POP (30 mcg levonogestrel) ó DIU de cobre. Hubo diferencias significativas 
en la discontinuación de los anticonceptivos por cualquier motivo $(35 \%, 55 \%, 29 \% ; p<0,01)$ y por motivos médicos $(11 \%, 31 \%, 4 \% ; p<0,05)$ en los grupos de ACO, POP y DIU, respectivamente. Las náuseas fueron más frecuentes en el grupo $\mathrm{ACO}$, el acné e hirsutismo en las usuarias de POP, y la dismenorrea en el grupo de DIU. No hubo cambios significativos en la presión arterial ni en el peso corporal. Este estudio concluyó que los efectos adversos no relacionados a la actividad lúpica no son razones frecuentes para discontinuar los métodos anticonceptivos, y que el uso de POP tiene menor adherencia que el uso de los otros métodos. Los autores de este estudio atribuyen la baja adherencia de las POP a las alteraciones en el patrón del flujo menstrual, y a "razones personales".

Las mujeres con LES tienen un riesgo de fractura 5 veces mayor en comparación a las sin esta patología. Esta diferencia es mayor en las mujeres entre 18 a 24 años (31). El uso de ACO en pacientes con LES se ha asociado a un menor riesgo de daño músculo-esquelético (atrofia muscular, osteoporosis con fractura o colapso vertebral, necrosis avascular, osteomielitis, rotura de tendón o artritis deformante (OR 0,48; IC95\% 0,28-0,81) (32), y a menor riesgo de fracturas (28).

Estudios observacionales no han encontrado asociación entre el uso de ACO en LES y el desarollo de enfermedad vascular periférica (33), nefritis lúpica o cáncer de mama (16), pero sí una mayor frecuencia de Papanicolaou anormal (34).
Criterios de elegibilidad para métodos anticonceptivos en LES. Se han elaborado distintas guías clínicas por grupos de expertos internacionales para el uso de anticoncepción en distintas situaciones clínicas. La World Health Organization Medical Eligibility Criteria for Contraceptive Use (WHO-MEC) ha publicado guías en torno al uso de anticoncepción en LES, basado en la evidencia actual disponible (26) (Tabla I). La Centers of Disease Control (CDS) (35) adaptó la WHO-MEC para el uso en la población de Estados Unidos en base a sus características y disponibilidad de métodos anticonceptivos, pero mantiene la mayoría de las sugerencias de la WHO-MEC. Sus principales conclusiones son que la mayoría de los métodos anticonceptivos se pueden indicar en mujeres con LES inactivo o activo estable. El parche transdérmico, el anillo vaginal y los métodos inyectables combinados son similares en su composición a los ACO, por lo que la OMS considera que las recomendaciones emitidas para este método, según la evidencia actual, son extrapolables a otras vías de administración. Las pacientes con anticuerpos antifosfolípidos positivos o desconocidos son categoría 4 para la OMS, es decir, está contraindicado el uso de $\mathrm{AH}$ combinado, y el uso de progestina sola es categoría 3, es decir, donde los riesgos superan a los beneficios del método. La existencia de otras comorbilidades y factores de riesgo cardiovasculares deben ser evaluadas en base a la guía OMS y al criterio médico.

Tabla I

\section{USO DE ANTICONCEPCIÓN EN PACIENTES CON LES ADAPTADO EN BASE} A LAS RECOMENDACIONES DE LA OMS (29)

\begin{tabular}{lcccc}
\hline & \multicolumn{1}{c}{$\begin{array}{c}\text { Métodos } \\
\text { combinados: } \\
\text { Oral, anillo, parche, } \\
\text { inyectable }\end{array}$} & $\begin{array}{c}\text { Mrales, implantes, } \\
\text { DIU con LNG }\end{array}$ & $\begin{array}{c}\text { Inyectables } \\
\text { (DMPA/NET-EN) }\end{array}$ & $\begin{array}{c}\text { DIU } \\
\text { con cobre }\end{array}$ \\
\hline $\begin{array}{l}\text { Anticuerpos antifosfolípidos } \\
\text { positivos o desconocido }\end{array}$ & 4 & 3 & 3 & 1 \\
$\begin{array}{l}\text { Trombocitopenia severa } \\
\text { Tratamiento inmunosupresivo }\end{array}$ & 2 & 2 & 3: Inicio & 3: Inicio \\
2: Continuación & 2: Continuación & 2: Inicio \\
Ninguno de los anteriores & 2 & 2 & 2 & 1: Continuación \\
\hline
\end{tabular}

Categoría 1: Condición en la cual no hay restricción para el uso del método anticonceptivo.

Categoría 2: Condición en donde las ventajas de usar el método generalmente superan las desventajas del uso del método.

Categoría 3: Condición donde los riesgos teóricos o probados superan las ventajas del uso del método.

Categoría 4: Condición que representa un riesgo inaceptable para la salud con el uso del método anticonceptivo.

DMPA: acetato de medroxiprogesterona de depósito/NET-EN: enantato de noretistenona/ DIU: Dispositivo intrauterino/ LNG: levonogestrel. 


\section{ARTRITIS REUMATOIDE Y ANTICONCEPCIÓN}

La artritis reumatoide (AR) es una enfermedad autoinmune de origen desconocido que afecta las articulaciones periféricas de manera simétrica. Generalmente la inflamación deriva a deformidad de tendones y ligamentos, y destrucción de las articulaciones por la erosión de cartílago y huesos. Se presenta más frecuentemente en mujeres con una relación de 2-3:1 con los hombres (36). La enfermedad tiende a disminuir su actividad durante el embarazo ya que es una condición mediada por citoquinas Th1, por lo que teóricamente la exposición a estrógenos disminuiría su actividad (1). La planificación reproductiva se hace necesaria, considerando el uso de medicamentos como el Metotrexate que posee efectos teratogénicos.

Actividad de AR y anticoncepción. Una RS reciente evaluó el uso de anticonceptivos en mujeres con AR (37). Incluyó 8 artículos: 6 con uso de ACO, 1 con POP, y 1 con estrógenos-solo. No se encontró evidencia para el uso de DIU o progesterona inyectable. El único estudio de mayor calidad metodológica (38) fue un estudio prospectivo de 112 mujeres entre 20 a 50 años, las cuales 54 usaron ACO (no se describe formulación) luego del diagnóstico vs 58 que no usaron. En el grupo usuario de ACO se observó una tendencia a la mejoría en los síntomas subjetivos y objetivos de AR, pero sin significancia estadística. Los otros estudios mostraron escaso o nulo efecto sobre la actividad de la enfermedad con el uso de anticoncepción. A pesar de la escasa evidencia, esta RS concluyó que el uso de $\mathrm{AH}$ no afecta la progresión de la actividad de AR.

Categorías de seguridad de distintos métodos anticonceptivos en AR. Actualmente la WHO-MEC no incluye a la $A R$ como una condición médica que contraindique el uso de AH. La CDS el año 2013 incluyó guías para el uso de anticonceptivos en pacientes con AR (Tabla II). Frente a la escasa evidencia actual, que no sugiere efectos adversos del uso de $\mathrm{AH}$ en pacientes con AR, hoy en día no existe contraindicación del uso de éstos métodos anticonceptivos en estas pacientes.

\section{OTRAS ENFERMEDADES REUMATOLÓGICAS Y ANTICONCEPCIÓN}

En otras ER, tales como síndrome de Sjöegren, dermatomiositis, o esclerosis sistémica, no se ha demostrado una asociación con la acción de hormonas sexuales femeninas. Existe escasa evidencia disponible, sin embargo, el uso de $\mathrm{AH}$ en estas mujeres probablemente es seguro, siempre y cuando se descarte la presencia de anticuerpos antifosfolípidos positivos. Se ha visto que en mujeres que presentan el fenómeno de Raynaud el estrógeno pudiera tener un efecto vasodilatador (39), y en pacientes con uso prolongado de corticoides el uso de $\mathrm{AH}$ combinada podría mejorar el índice de mineralización ósea (40).

\section{INTERACCIONES FARMACOLÓGICAS ENTRE AH Y ANTIRREUMÁTICOS}

Existe escasa evidencia de las interacciones farmacológicas entre el uso de $\mathrm{AH}$ y medicamentos de la esfera reumatológica. No se ha encontrado interacción entre el uso de AH y prednisona, AINES, ciclofosfamida, metotrexate, cloroquina, azatriopina o rituximab. En cuanto al micofenolato, se ha visto que disminuye el efecto del etinilestradiol, por lo que se recomienda monitorizar y/o usar método alternativo. También el micofenolato disminuye la

\section{Tabla II \\ USO DE ANTICONCEPCIÓN EN PACIENTES CON AR ADAPTADO EN BASE A LAS RECOMENDACIONES DE LA CDC (35)}

\begin{tabular}{|c|c|c|c|c|c|}
\hline \multirow[b]{2}{*}{ Condición } & \multirow{2}{*}{$\begin{array}{c}\text { Métodos } \\
\text { combinados: } \\
\text { Oral, anillo, parche }\end{array}$} & \multicolumn{3}{|c|}{ Métodos de progestina sola: } & \multirow[b]{2}{*}{ DIU con cobre } \\
\hline & & $\begin{array}{c}\text { Orales, } \\
\text { implantes. }\end{array}$ & $\begin{array}{l}\text { Inyectables } \\
\text { (DMPA) }\end{array}$ & DIU con LNG & \\
\hline $\begin{array}{l}\text { En tratamiento } \\
\text { inmunosupresivo }\end{array}$ & 2 & 1 & $2 / 3$ & $\begin{array}{c}\text { 2: Inicio } \\
\text { 1: Continuación }\end{array}$ & $\begin{array}{c}\text { 2: Inicio } \\
\text { 1: Continuación }\end{array}$ \\
\hline $\begin{array}{l}\text { Sin tratamiento } \\
\text { inmunosupresivo }\end{array}$ & 2 & 1 & 2 & 1 & 1 \\
\hline
\end{tabular}

Categoría 1: Condición en la cual no hay restricción para el uso del método anticonceptivo.

Categoría 2: Condición en donde las ventajas de usar el método generalmente superan las desventajas del uso del método.

Categoría 3: Condición donde los riesgos teóricos o probados superan las ventajas del uso del método.

Categoría 4: Condición que representa un riesgo inaceptable para la salud con el uso del método anticonceptivo. DMPA: acetato de medroxiprogesterona de depósito/ DIU: Dispositivo intrauterino/ LNG: levonogestrel. 
efectividad de levonogestrel, por lo que se sugiere monitorizar y/o utilizar mecanismo anticonceptivo de barrera adicional.

\section{CONCLUSIONES}

Con la evidencia actual muchas mujeres con $E R$, incluido LES, pueden ser consideradas candidatas para el uso de $\mathrm{AH}$ como un método seguro para el manejo de metrorragia, dismenorrea y planificación familiar. El beneficio del uso de $\mathrm{AH}$ y la prevención del embarazo supera los riesgos asociados al uso de AH combinado en muchas de ellas. Pacientes con LES inactivo o con actividad leve a moderada podrían recibir cualquier tipo de $\mathrm{AH}$ mientras se descarte la presencia de anticuerpos antifosfolípidos positivos, al igual que familiares de pacientes con ER que requieran $\mathrm{AH}$. Pacientes con AR no presentan contraindicación del uso de AH por no haber cambios demostrados sobre la actividad de la enfermedad, al igual que en otras ER. La escasa interacción farmacológica entre el uso de $\mathrm{AH}$ y medicamentos reumatológicos, permite contar con seguridad en el uso de métodos anticonceptivos en este grupo de mujeres. Aún la evidencia del uso de $\mathrm{AH}$ en mujeres menores de 18 años con patologías reumatológicas es escasa.

\section{REFERENCIAS}

1. Nalbandian G, Kovats S. Estrogen, immunity and autoimmune disease. Curr Med Chem-Immunol Endocr Metab Agents 2005;5:85-91.

2. Roubinian JR, Talal N, Greenspan JS, Goodman JR, Siiteri PK. Delayed androgen treatment prolongs survival in murine lupus. J Clin Invest 1979;63:902-11.

3. Bynoe MS, Grimaldi CM, Diamond B. Estrogen up-regulates $\mathrm{Bcl}-2$ and blocks tolerance induction of naïve B cells. Proc Natl Acad Sci USA 2000;97:2703-8.

4. Sánchez-Guerrero J, Karlson EW, Liang MH, Hunter DJ, Speizer FE, Colditz GA. Past use of oral contraceptives and the risk of developing systemic lupus erythematosus. Arthritis Rheum 1997;40:804-8.

5. McCarty DJ, Manzi S, Medsger TA Jr, Ramsey-Goldman R, LaPorte RE, Kwoh CK. Incidence of systemic lupus erythematosus. Race and gender differences. Arthritis Rheum 1995;38:1260-70.

6. Masi AT, Kaslow RA. Sex effects in systemic lupus erythematosus: a clue to pathogenesis. Arthritis Rheum 1978;21:480-4.

7. Carmona F, Font J, Cervera R, Muñoz F, Cararach $\mathrm{V}$, Balasch J. Obstetrical outcome of pregnancy in patients with systemic lupus erythematosus. A study of 60 cases. Eur J Obstet Gynecol Reprod Biol 1999:83:137-42.

8. Petri M. Hopkins Lupus Pregnancy Center: 1987 to 1996. Rheum Dis Clin North Am 1997;23:1-13.

9. Clowse ME, Magder LS, Witter F, Petri M. The impact of increased lupus activity on obstetric outcomes. Arthritis Rheum 2005;52:514-21.
10. Cortes-Hernandez J, Ordi-Ros J, Paredes F, Casellas M, Castillo F, Vilardell-Torres M. Clinical predictors of fetal and maternal outcome in systemic lupus erythematosus: a prospective study of 103 pregnancies. Rheumatology (Oxford) 2002;41:643-50.

11. Bombardier C, Gladman DD, Urowitz MB, Caron D, Chang $\mathrm{CH}$. Derivation of SLEDAl. A disease activity index for lupus patients. The Committee of prognosis studies in SLE. Arthritis Rheum 1992;35:630-40.

12. Barr SG, Zonana-Nacach A, Magder LS, Petri M. Patterns of disease activity in systemic lupus erythematosus. Arthritis Rheum 1999;42:2682-8.

13. Jungers $P$, Dougados $M$, Pelissier $C$, Kuttenn F, Tron $F$, Lesavre $P$, et al. Influence of oral contraceptive therapy on the activity of systemic lupus erythematosus. Arthritis Rheum 1982;25:618-23.

14. Julkunen HA. Oral contraceptives in systemic lupus erythematosus: side-effects and influence on the activity of SLE. Scand J Rheumatol 1991;20:427-33.

15. Petri M, Kim MY, Kalunian KC, et al. Combined oral contraceptives in women with systemic lupus erythematosus. N Engl J Med 2005;353:2550-8.

16. Sánchez-Guerrero J, Uribe AG, Jiménez-Santana $\mathrm{L}$, et al. A trial of contraceptive methods in women with systemic lupus erythematosus. $\mathrm{N}$ Engl $\mathrm{J}$ Med 2005;353:2539-49.

17. Culwell KR, Curtis KM, del Carmen Cravioto M. Safety of contraceptive method use among women with systemic lupus erythematosus: a systematic review. Obstet Gynecol 2009;114:341-53.

18. Venous thromboembolic disease and combined oral contraceptives: results of international multicentre case-control study: WHO Collaborative Study of Cardiovascular Disease and Steroid Hormone Contraception. Lancet 1995;346:1575-82.

19. Vandenbroucke JP, Rosing J, Bloemenkamp KW, Middeldorp S, Helmerhorst FM, Bouma BN, et al. Oral contraceptives and the risk of venous thrombosis. $\mathrm{N}$ Engl J Med 2001;344:1527-35.

20. Gordon C, Pitts S. Approach to the adolescent requesting contraception. J Clin Endocrinol Metab 2012;97:9-15.

21. Vandenbroucke JP, Rosing J, Bloemenkamp KW, Middeldorp S, Helmerhorst FM, Bouma BN, Rosendaal FR. Oral contraceptives and the risk of venous thrombosis, N Eng J Med 2001;344:1527-35.

22. Sarabi ZS, Chang E, Bobba R, Ibanez D, Gladman D, Urowitz $\mathrm{M}$, et al. Incidence rates of arterial and venous thrombosis after diagnosis of systemic lupus erythematosus. Arthritis Rheum 2005;53:609-12.

23. Romero-Díaz J, García-Sosa I, Sánchez-Guerrero J. Thrombosis in systemic lupus erythematosus and other autoimmune diseases of recent onset. J Rheumatol 2009;36:68-75.

24. HW Li R, Gebbie A, Wong R et al. The use of sex hormones in woman with rheumatological disease. Hong Kong Med J 2011;17:487-91.

25. Somers E, Magder LS, Petri M. Antiphospholipid antibodies and incidence of venous thrombosis in a cohort of patients with systemic lupus erythematosus. J Rheumatol 2002;29:2531-6. 
26. Wahl DG, Guillemin F, de Maistre E, Perret C, Lecompte T, Thibaut G. Risk for venous thrombosis related to antiphospholipid antibodies in systemic lupus erythematosus-a meta-analysis. Lupus 1997;6:46773.

27. Chopra N, Koren S, Greer WL, Fortin PR, Rauch J, Fortin I, et al. Factor $\mathrm{V}$ Leiden, prothrombin gene mutation, and thrombosis risk in patients with antiphospholipid antibodies. J Rheumatol 2002;29:1683-8.

28. Choojitarom K, Verasertniyom O, Totemchokchyakarn K, Nantiruj K, Sumethkul V, Janwityanujit S. Lupus nephritis and Raynaud's phenomenon are significant risk factors for vascular thrombosis in SLE patients with positive antiphospholipid antibodies. Clin Rheumatol 2008;27:345-51.

29. World Health Organization. Medical eligibility criteria for contraceptive use. 4th ed. Geneva: World Health Organization; 2009.

30. Cravioto MC, Jiménez-Santana L, Mayorga J, Seuc A. Side effects unrelated to disease activity and acceptability of highly effective contraceptive methods in women with systemic lupus erythematosus: a randomized, clinical trial. Contraception 2014;90:147-53.

31. Ramsey-Goldman R, Dunn JE, Huang CF, Dunlop D, Rairie JE, Fitzgerald S, et al. Frequency of fractures in women with systemic lupus erythematosus: comparison with United States population data. Arthritis Rheum 1999;42:882-90.

32. Petri M. Musculoskeletal complications of systemic lupus erythematosus in the Hopkins Lupus Cohort: an update. Arthritis Care Res 1995;8:137-45.
33. McDonald J, Stewart J, Urowitz MB, Gladman DD. Peripheral vascular disease in patients with systemic lupus erythematosus. Ann Rheum Dis 1992;51:56-60.

34. Bernatsky S, Ramsey-Goldman R, Gordon C, Joseph L, Boivin JF, Rajan R, et al. Factors associated with abnormal Pap results in systemic lupus erythematosus. Rheumatology 2004;43:1386-9.

35. Centers of Disease Control and prevention. US. Medical Eligibility Criteria for Contraceptive Use. MMWR 2013;62(RR05);1-46. Disponible en: http://www.cdc. $\mathrm{gov} / \mathrm{mmwr} / \mathrm{preview} / \mathrm{mmwrhtml} / \mathrm{rr6205a1}$.htm

36. Helmick CG, Felson DT, Lawrence RC, et al. Estimates of the prevalence of arthritis and other rheumatic conditions in the United States, Part I. Arthritis Rheum 2008;58:15-25.

37. Farr S, Folger S, Paulen M, Curtis K. Safety of contraceptive methods for women with rheumatoid arthritis: a systematic review. Contraception 2010;82:64-71.

38. Drossaers-Bakker KW, Zwinderman AH, Van ZD, Breedveld FC, Hazes JM. Pregnancy and oral contraceptive use do not significantly influence outcome in long term rheumatoid arthritis. Ann Rheum Dis 2002;61:405-8.

39. Generini S, Seibold JR, Matucci-Cerinic M. Estrogens and neuropeptides in Raynaud's phenomenon. Rheum Dis Clin North Am 2005;31:177-86, x-xi.

40. Gourlay M, Franceschini N, Sheyn Y. Prevention and treatment strategies for glucocorticoid-induced osteoporotic fractures. Clin Rheumatol 2007;26:144-53. 